UB-ECM-PF 01/11

DESY 01-120

NYU-TH/01/08/09

hep-ph/0109228

September 2001

\title{
Width and Partial Widths of Unstable Particles in the Light of the Nielsen Identities
}

\author{
Pietro A. Grassi, ${ }^{1}$ Bernd A. Kniehl, ${ }^{2 *}$ Alberto Sirlin $^{1}$ \\ ${ }^{1}$ Department of Physics, New York University, \\ 4 Washington Place, New York, NY 10003, USA \\ 2 Departament d'Estructura i Constituents de la Matèria, Universitat de Barcelona, \\ Avinguda Diagonal, 647, 08028 Barcelona, Spain
}

\begin{abstract}
Fundamental properties of unstable particles, including mass, width, and partial widths, are examined on the basis of the Nielsen identities (NI) that describe the gauge dependence of Green functions. In particular, we prove that the pole residues and associated definitions of branching ratios and partial widths are gauge independent to all orders. A simpler, previously discussed definition of branching ratios and partial widths is found to be gauge independent through next-to-nextto-leading order. It is then explained how it may be modified in order to extend the gauge independence to all orders. We also show that the physical scattering amplitude is the most general combination of self-energy, vertex, and box contributions that is gauge independent for arbitrary $s$, discuss the analytical properties of the NI functions, and exhibit explicitly their one-loop expressions in the $Z$ - $\gamma$ sector of the Standard Model.
\end{abstract}

PACS numbers: 11.10.Gh, 11.15.-q

*Permanent address: II. Institut für Theoretische Physik, Universität Hamburg, Luruper Chaussee 149, 22761 Hamburg, Germany. 


\section{INTRODUCTION}

The conventional definitions of the mass and width of unstable particles are

$$
\begin{aligned}
& M^{2}=M_{0}^{2}+\operatorname{Re} A\left(M^{2}\right), \\
& M \Gamma=-\frac{\operatorname{Im} A\left(M^{2}\right)}{1-\operatorname{Re} A^{\prime}\left(M^{2}\right)},
\end{aligned}
$$

where $M_{0}$ is the bare mass and $A(s)$ is the self-energy in the case of scalar bosons and the transverse self-energy in the case of vector bosons. The partial widths are defined by expressing the numerator of Eq. (2) as a sum of cut contributions involving distinct sets of final-state physical particles. We will refer to $M$ as the on-shell mass and to Eqs. (1) and (2) as the conventional on-shell formulation.

However, it was shown in Ref. [1] that, in a gauge theory, Eqs. (11) and (2) become gauge dependent in next-to-next-to-leading order (NNLO), i.e. in $O\left(g^{4}\right)$ and $O\left(g^{6}\right)$, respectively, where $g$ is a generic gauge coupling. In the same papers, it was proposed that a way of solving this problem is to base the definitions of mass and width on the complex-valued position of the propagator's pole:

$$
\bar{s}=M_{0}^{2}+A(\bar{s}) .
$$

Employing the parameterization $\bar{s}=m_{2}^{2}-i m_{2} \Gamma_{2}$ of Ref. [1], we have

$$
\begin{aligned}
m_{2}^{2} & =M_{0}^{2}+\operatorname{Re} A(\bar{s}), \\
m_{2} \Gamma_{2} & =-\operatorname{Im} A(\bar{s}) .
\end{aligned}
$$

From Eq. (㺼), we see that the mass counterterm is given by $\operatorname{Re} A(\bar{s})$, rather than $\operatorname{Re} A\left(M^{2}\right)$.

In the recent past, a number of authors have advocated definitions of mass and width based on $\bar{s}$ [2], and the conclusions of Ref. [1] have been confirmed by later studies [3, 4] and proven to all orders [5]. In particular, it has been shown that the gauge dependences of $M$ and $\Gamma$ are numerically large in the case of a heavy Higgs boson $\mathbb{1}$. It has also been pointed out that Eq. (2) leads to serious problems if $A(s)$ is not analytic in the neighborhood of $M^{2}$, a situation that occurs when $M$ is very close to a physical threshold [6] or, in the resonance region, when the unstable particle is coupled to massless quanta, as in the cases of the $W$ boson and unstable quarks [7].

If Eq. (5) is a consistent definition of width, an important question naturally arises: what is the definition of partial widths? A recent analysis of that concept, with special emphasis on issues of gauge independence and additivity, was given in Ref. [8].

The aim of the present paper is to revisit the important problem of width and partial widths of unstable particles in the light of the Nielsen identities (NI) [9, 10, 11, 12, 13, Since the variation of Green functions with respect to gauge parameters can be viewed as Becchi-Rouet-Stora-Tyutin (BRST) transformations, it is convenient to enlarge the BRST symmetry to include also the gauge parameters themselves. In this new framework, known

as extended BRST symmetry, a particular set of Slavnov-Taylor identities [14], which are 
frequently called NI, describe the gauge dependence of Green functions [9, 10, 11, 12, 13]. (Originally, the NI were formulated for one-particle-irreducible (1PI) Green functions in connection with the effective scalar potential in a special class of gauges.) In Sec. II, we employ the NI to show that $M$ and $\Gamma$, defined by Eqs. (11) and (2), are indeed gauge dependent in $O\left(g^{4}\right)$ and $O\left(g^{6}\right)$, respectively. For completeness, we also briefly review the proof that $\bar{s}$ is gauge independent [5]. In Sec. III, we apply the NI to understand and clarify several of the results and theoretical issues presented in Ref. [8]. In particular, we show that a definition of branching ratios and partial widths based on the pole residues is gauge independent to all orders, while a simpler, previously discussed formulation is gauge independent through NNLO. In Sec. IV, we explain how the simpler definition can be modified to extend the gauge independence to all orders. In Sec. V, we discuss the NI for box diagrams and show that the physical amplitude is the most general combination of self-energy, vertex, and box contributions that is gauge independent for all values of $s$. Section VI deals with the analytical properties of the NI functions, and the Appendix presents their one-loop expressions in the $Z-\gamma$ sector of the Standard Model (SM).

\section{NI FOR $\Pi\left(s, \xi_{k}\right)$ AND GAUGE DEPENDENCE OF $M$ AND $M \Gamma$}

The transverse propagator of a gauge field is of the form

$$
\mathcal{D}_{\mu \nu}=-i \frac{Q_{\mu \nu}}{\Pi\left(s, \xi_{k}\right)},
$$

where $Q_{\mu \nu}=g_{\mu \nu}-p_{\mu} p_{\nu} / s, p_{\mu}$ is the four-momentum, $s=p^{2}$, and $\xi_{k}$ is a generic gauge parameter. In the absence of mixing, we have

$$
\Pi\left(s, \xi_{k}\right)=s-M_{0}^{2}-A\left(s, \xi_{k}\right)=s-m_{2}^{2}-\operatorname{Re}\left[A\left(s, \xi_{k}\right)-A\left(\bar{s}, \xi_{k}\right)\right]-i \operatorname{Im} A\left(s, \xi_{k}\right) .
$$

In the last expression, $M_{0}^{2}$ has been expressed in terms of $m_{2}^{2}$ via Eq. (四).

The NI for $\Pi\left(s, \xi_{k}\right)$ reads

$$
\frac{\partial}{\partial \xi_{l}} \Pi\left(s, \xi_{k}\right)=2 \Lambda_{l}\left(s, \xi_{k}\right) \Pi\left(s, \xi_{k}\right),
$$

where $\Lambda_{l}\left(s, \xi_{k}\right)$ is a complex, amputated, 1PI, two-point Green function of $O\left(g^{2}\right)$ involving the gauge field, its BRST variation, and the gauge fermion (see, e.g., Eq. (42) of Ref. [5], Eq. (2.15) of Ref. [10], Eq. (4.9a) of Ref. [11], and Eq. (21) of Ref. [13]). We recall that the sum of the gauge-fixing and ghost terms in the Lagrangian density can be expressed as the BRST variation of the gauge fermion, and that the latter is coupled to the BRST variation of the gauge parameter $\xi_{l}$. It is understood that the vertex corresponding to the BRST variation of $\xi_{l}$ carries zero momentum, so that $\Lambda_{l}\left(s, \xi_{k}\right)$ depends kinematically only on $s$. The simple form of Eq. (8) can only be achieved by a proper treatment of 
the Higgs tadpole: either one chooses to renormalize the one-point function to cancel the tadpole contribution or to reabsorb it into a redefinition of the self-energy $\Pi\left(s, \xi_{k}\right)$ [5, 15].

Eq. (8) permits one to immediately understand the gauge independence of $\bar{s}$ [5]. As $\bar{s}$ is the zero of $\Pi\left(s, \xi_{k}\right)$, we have

$$
\Pi\left(\bar{s}, \xi_{k}\right)=0 .
$$

Differentiating Eq. (9) with respect to $\xi_{l}$, we obtain

$$
\frac{\partial \bar{s}}{\partial \xi_{l}} \frac{\partial}{\partial \bar{s}} \Pi\left(\bar{s}, \xi_{k}\right)+\frac{\partial}{\partial \xi_{l}} \Pi\left(\bar{s}, \xi_{k}\right)=0 .
$$

However, Eqs. (8) and (9) tell us that the second term in Eq. (10) vanishes. Since $\partial \Pi\left(\bar{s}, \xi_{k}\right) / \partial \bar{s}=1+O\left(g^{2}\right)$, we find

$$
\frac{\partial \bar{s}}{\partial \xi_{l}}=0
$$

the result of Ref. [5].

Instead, taking the real part of Eq. (8), we have

$$
\frac{\partial}{\partial \xi_{l}} \operatorname{Re} \Pi\left(s, \xi_{k}\right)=2 \operatorname{Re} \Lambda_{l}\left(s, \xi_{k}\right) \operatorname{Re} \Pi\left(s, \xi_{k}\right)-2 \operatorname{Im} \Lambda_{l}\left(s, \xi_{k}\right) \operatorname{Im} \Pi\left(s, \xi_{k}\right) .
$$

By definition, the on-shell mass is the zero of $\operatorname{Re} \Pi\left(s, \xi_{k}\right)$. Thus,

$$
\operatorname{Re} \Pi\left(M^{2}, \xi_{k}\right)=0
$$

Differentiating Eq. (13) with respect to $\xi_{l}$ and using Eq. (12), we have

$$
\frac{\partial M^{2}}{\partial \xi_{l}} \operatorname{Re} \Pi^{\prime}\left(M^{2}, \xi_{k}\right)-2 \operatorname{Im} \Lambda_{l}\left(M^{2}, \xi_{k}\right) \operatorname{Im} \Pi\left(M^{2}, \xi_{k}\right)=0
$$

where the prime indicates a derivative with respect to the first argument. Eq. (14) leads to

$$
\frac{\partial M^{2}}{\partial \xi_{l}}=2 \frac{\operatorname{Im} \Lambda_{l}\left(M^{2}, \xi_{k}\right) \operatorname{Im} \Pi\left(M^{2}, \xi_{k}\right)}{\operatorname{Re} \Pi^{\prime}\left(M^{2}, \xi_{k}\right)} .
$$

Since $\operatorname{Re} \Pi^{\prime}\left(M^{2}, \xi_{k}\right)=O(1)$ and both $\operatorname{Im} \Lambda_{l}\left(M^{2}, \xi_{k}\right)$ and $\operatorname{Im} \Pi\left(M^{2}, \xi_{k}\right)$ are of $O\left(g^{2}\right)$,

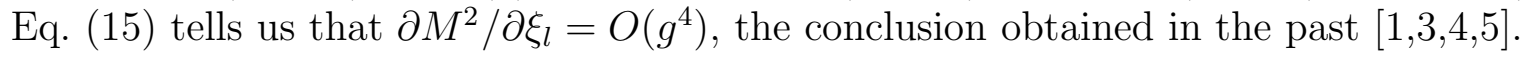

Turning our attention to Eq. (2), we note that it may be written as

$$
M \Gamma=\frac{\operatorname{Im} \Pi\left(M^{2}, \xi_{k}\right)}{\operatorname{Re} \Pi^{\prime}\left(M^{2}, \xi_{k}\right)} .
$$

The imaginary part of Eq. (8) tells us that

$$
\frac{\partial}{\partial \xi_{l}} \operatorname{Im} \Pi\left(M^{2}, \xi_{k}\right)=2 \operatorname{Re} \Lambda_{l}\left(M^{2}, \xi_{k}\right) \operatorname{Im} \Pi\left(M^{2}, \xi_{k}\right),
$$


while, from Eqs. (12) and (13), we see that

$$
\frac{\partial}{\partial \xi_{l}} \operatorname{Re} \Pi^{\prime}\left(M^{2}, \xi_{k}\right)=2 \operatorname{Re} \Lambda_{l}\left(M^{2}, \xi_{k}\right) \operatorname{Re} \Pi^{\prime}\left(M^{2}, \xi_{k}\right)-2\left[\operatorname{Im} \Lambda_{l}\left(M^{2}, \xi_{k}\right) \operatorname{Im} \Pi\left(M^{2}, \xi_{k}\right)\right]^{\prime} .
$$

Combining Eqs. (17) and (18), we have

$$
\frac{\partial}{\partial \xi_{l}} \frac{\operatorname{Im} \Pi\left(M^{2}, \xi_{k}\right)}{\operatorname{Re} \Pi^{\prime}\left(M^{2}, \xi_{k}\right)}=2 \frac{\operatorname{Im} \Pi\left(M^{2}, \xi_{k}\right)\left[\operatorname{Im} \Lambda_{l}\left(M^{2}, \xi_{k}\right) \operatorname{Im} \Pi\left(M^{2}, \xi_{k}\right)\right]^{\prime}}{\left[\operatorname{Re} \Pi^{\prime}\left(M^{2}, \xi_{k}\right)\right]^{2}}
$$

To obtain the total derivative, we add the term $\left(\partial M^{2} / \partial \xi_{l}\right) \partial\left[\operatorname{Im} \Pi\left(M^{2}\right) / \operatorname{Re} \Pi^{\prime}\left(M^{2}\right)\right] / \partial M^{2}$ and find that

$$
\frac{d}{d \xi_{l}} \frac{\operatorname{Im} \Pi\left(M^{2}\right)}{\operatorname{Re} \Pi^{\prime}\left(M^{2}\right)}=2 \frac{\left\{\operatorname{Im} \Lambda_{l}\left(M^{2}\right)\left[\operatorname{Im} \Pi\left(M^{2}\right)\right]^{2}\right\}^{\prime}}{\left[\operatorname{Re} \Pi^{\prime}\left(M^{2}\right)\right]^{2}}-2 \frac{\operatorname{Im} \Lambda_{l}\left(M^{2}\right)\left[\operatorname{Im} \Pi\left(M^{2}\right)\right]^{2} \operatorname{Re} \Pi^{\prime \prime}\left(M^{2}\right)}{\left[\operatorname{Re} \Pi^{\prime}\left(M^{2}\right)\right]^{3}} .
$$

In Eq. (20) and henceforth, we do not explicitly display the dependence of the various Green functions on $\xi_{k}$.

Since $\operatorname{Im} \Lambda_{l}\left(M^{2}\right), \operatorname{Im} \Pi\left(M^{2}\right)$, and $\operatorname{Re} \Pi^{\prime \prime}\left(M^{2}\right)$ are of $O\left(g^{2}\right)$ and $\operatorname{Re} \Pi^{\prime}\left(M^{2}\right)=O(1)$, in leading order, Eq. (20) reduces to

$$
\frac{d}{d \xi_{l}} \frac{\operatorname{Im} \Pi\left(M^{2}\right)}{\operatorname{Re} \Pi^{\prime}\left(M^{2}\right)}=2\left\{\operatorname{Im} \Lambda_{l}\left(M^{2}\right)\left[\operatorname{Im} \Pi\left(M^{2}\right)\right]^{2}\right\}^{\prime}+O\left(g^{8}\right) .
$$

Thus, we see that the conventional definition of width [Eq. (2)] is gauge dependent in NNLO, i.e. in $O\left(g^{6}\right)$, in agreement with the conclusion of Refs. [1, 3, 4, 5].

An important implication of the above results is that, in a gauge theory, Eqs. (四) and (2) can be identified with the physical observables only through next-to-leading order (NLO).

In the case of mixing between two fields $A$ and $B$, Eq. (8) is replaced by

$$
\frac{\partial}{\partial \xi_{l}} \Pi_{\alpha \beta}(s)=\sum_{\delta}\left[\Lambda_{l, \alpha}^{\delta}(s) \Pi_{\delta \beta}(s)+\Lambda_{l, \beta}^{\delta}(s) \Pi_{\delta \alpha}(s)\right]
$$

where $\alpha, \beta, \delta=A, B$, the diagonal elements $\Pi_{\alpha \alpha}(s) \equiv s-M_{0, \alpha}^{2}-A_{\alpha \alpha}(s)$ are the analogues of Eq. (7), and, for $\alpha \neq \beta, \Pi_{\alpha \beta}(s) \equiv-A_{\alpha \beta}(s)$, with $A_{\alpha \beta}(s)$ being the mixed $A$ - $B$ selfenergy (we use the sign conventions of Ref. [15]). Here, $\Lambda_{l, \alpha}^{\delta}(s)$ are mixed two-point functions involving the field $\alpha$, the BRST variation of $\xi_{l}$, and the source of the BRST variation of the field $\delta$. Correspondingly, the pole positions $\bar{s}_{A}$ and $\bar{s}_{B}$ are the zeroes of

$$
D(s)=\Pi_{A A}(s) \Pi_{B B}(s)-\Pi_{A B}^{2}(s)
$$

Using Eq. (22), one finds

$$
\frac{\partial}{\partial \xi_{l}} D(s)=2\left[\Lambda_{l, A}^{A}(s)+\Lambda_{l, B}^{B}(s)\right] D(s)
$$


and, therefore,

$$
\frac{\partial}{\partial \xi_{l}} D(\bar{s})=0
$$

Differentiating $D(\bar{s})=0$ with respect to $\xi_{l}$ and employing Eq. (25), one obtains again the result $\partial \bar{s} / \partial \xi_{l}=0[5]$.

The transverse propagator of the field $A$ is

$$
-i \frac{\Pi_{B B}(s)}{D(s)}=\frac{-i}{s-M_{0, A}^{2}-A(s)},
$$

where

$$
A(s)=A_{A A}(s)+\frac{A_{A B}^{2}(s)}{\Pi_{B B}(s)} .
$$

We also note that Eqs. (22) and (24) lead to

$$
\frac{\partial}{\partial \xi_{l}} \frac{D(s)}{\Pi_{B B}(s)}=2\left[\Lambda_{l, A}^{A}(s)-\frac{\Lambda_{l, B}^{A}(s) \Pi_{A B}(s)}{\Pi_{B B}(s)}\right] \frac{D(s)}{\Pi_{B B}(s)} .
$$

\section{NI FOR VERTEX FUNCTIONS AND GAUGE PROPERTIES OF POLE RESIDUES AND PAR- TIAL WIDTHS}

We consider the amplitude $i \rightarrow Z \rightarrow f$, where $Z$ is an unstable gauge boson, and $i$ and $f$ are initial and final states, respectively, involving on-shell particles that are either stable or have negligible widths.

Using Eq. (3), we may express Eq. (6) as

$$
\mathcal{D}_{\mu \nu}=-i \frac{Q_{\mu \nu}}{s-\bar{s}-A(s)+A(\bar{s})} .
$$

The vertex amplitude defined by $Z$ and $f$ is given by

$$
V_{f}^{\mu}(s) \equiv\left\langle f\left|J_{Z}^{\mu}\right| 0\right\rangle=\sum_{a} v_{f}^{(a)}(s, \ldots) M_{f}^{(a) \mu}
$$

where $M_{f}^{(a) \mu}$ denote various independent vector and axial-vector matrix elements involving the spinors, polarization four-vectors, and four-momenta of the final-state particles, while $v_{f}^{(a)}(s, \ldots)$ are scalar functions. Here, it is understood that the amplitude $V_{f}^{\mu}(s)$ is not 1PI, but includes the field renormalizations of the external, on-shell particles in the state $f$. The dots indicate their dependence on the additional invariants constructed from the particles' momenta. We use the convention of including the generic coupling $g$ in the definition of $v_{f}^{(a)}(s, \ldots)$, so that, in leading order, $v_{f}^{(a)}(s, \ldots)=O(g)$. Expanding $V_{f}^{\mu}(s)$ and the denominator of Eq. (29) about $s=\bar{s}$, the overall amplitude may be expressed as

$$
\mathcal{A}_{f i}(s)=-i \frac{Q_{\mu \nu} V_{f}^{\mu}(\bar{s}) V_{i}^{\nu}(\bar{s})}{(s-\bar{s})\left[1-A^{\prime}(\bar{s})\right]}+N_{f i},
$$


where $N_{f i}$ stands for non-resonant contributions and, henceforth, we do not indicate the dependence on the additional invariants.

In the absence of mixing and using the extended BRST formalism, it is possible to derive the following identity for the gauge dependence of $V_{f}^{\mu}(s)$ :

$$
\frac{\partial}{\partial \xi_{l}} V_{f}^{\mu}(s)=\Lambda_{l}(s) V_{f}^{\mu}(s)+\Pi(s) \Delta_{l, f}^{\mu}(s),
$$

where $\Delta_{l, f}^{\mu}(s)$ is a complex Green function depending on the scalar invariants and involving the gauge field $Z$, the gauge fermion, and the sources for the fields of the final state $f$ (see, e.g., Eq. (45) of Ref. [5], Eq. (4.9b) of Ref. [11], and Eq. (14) of Ref. [13]). For instance, in a four-fermion amplitude, $f$ coincides with the two outgoing on-shell fermions, and, therefore, $\Delta_{l, f}^{\mu}(s)$ involves the latter. Although, as discussed after Eq. (8), the function $\Lambda_{l}\left(s, \xi_{k}\right)$ is a $1 \mathrm{PI}$ Green function, the new Green function $\Delta_{l, f}^{\mu}(s)$ is of the same nature as $V_{f}^{\mu}(s)$. It is functionally independent of $\Lambda_{l}(s)$ and incorporates the gauge dependence of the vertex $V_{f}^{\mu}(s)$ that is not proportional to the vertex itself. In leading order, $\Delta_{l, f}^{\mu}(s)=O\left(g^{3}\right)$. It is important to emphasize that the function $\Delta_{l, f}^{\mu}(s)$ in Eq. (32) does not contain contributions proportional to $1 / \Pi(s)$ and, in fact, is not singular at $s=\bar{s}$.

Differentiating Eq. (8) with respect to $s$ and setting $s=\bar{s}$, we have

$$
\frac{\partial}{\partial \xi_{l}} \Pi^{\prime}(\bar{s})=2 \Lambda_{l}(\bar{s}) \Pi^{\prime}(\bar{s})
$$

while Eq. (32) leads to

$$
\frac{\partial}{\partial \xi_{l}} V_{f}^{\mu}(\bar{s})=\Lambda_{l}(\bar{s}) V_{f}^{\mu}(\bar{s})
$$

Combining Eqs. (33) and (34), and recalling that $\bar{s}$ is $\xi_{l}$ independent, we obtain

$$
\frac{d}{d \xi_{l}} \frac{V_{f}^{\mu}(\bar{s})}{\sqrt{1-A^{\prime}(\bar{s})}}=0
$$

for any choice of $f$, a result that implies the gauge independence of the pole residues in Eq. (31). This is expected on general grounds [1,22, since such amplitudes are the residues of poles in the analytically continued scattering $(S)$ matrix. On the other hand, Eq. (35) provides a formal proof of this important conclusion.

In the case of mixing between two fields $A$ and $B$, Eq. (32) is generalized to

$$
\frac{\partial}{\partial \xi_{l}} \Gamma_{\alpha, f}^{\mu}(s)=\sum_{\delta}\left[\Lambda_{l, \alpha}^{\delta}(s) \Gamma_{\delta, f}^{\mu}(s)+\Pi_{\alpha \delta}(s) \Delta_{l, f}^{\delta, \mu}(s)\right],
$$

where $\alpha, \delta=A, B$ and $\Gamma_{\delta, f}^{\mu}(s)$ stand for vertex amplitudes that, again, include the field renormalizations of the external, on-shell particles. However, in the mixing case, the relevant vertex parts are combinations of the form

$$
V_{A, f}^{\mu}(s)=\Gamma_{A, f}^{\mu}(s)-\frac{\Pi_{A B}(s) \Gamma_{B, f}^{\mu}(s)}{\Pi_{B B}(s)} .
$$


For instance, in the neutral-current amplitude of the SM, the second term in Eq. (37) corresponds to one-particle-reducible contributions to the $Z^{0} f \bar{f}$ vertex arising from the $Z-\gamma$ mixing. Eqs. (22), (36), and (37) lead to

$$
\frac{\partial}{\partial \xi_{l}} V_{A, f}^{\mu}(s)=\left[\Lambda_{l, A}^{A}(s)-\frac{\Lambda_{l, B}^{A}(s) \Pi_{A B}(s)}{\Pi_{B B}(s)}\right] V_{A, f}^{\mu}(s)+\frac{D(s)}{\Pi_{B B}(s)}\left[\Delta_{l, f}^{A, \mu}(s)-\frac{\Lambda_{l, B}^{A}(s) \Gamma_{B, f}^{\mu}(s)}{\Pi_{B B}(s)}\right] .
$$

Setting $s=\bar{s}$, Eq. (38) reduces to

$$
\frac{\partial}{\partial \xi_{l}} V_{A, f}^{\mu}(\bar{s})=\left[\Lambda_{l, A}^{A}(\bar{s})-\frac{\Lambda_{l, B}^{A}(\bar{s}) \Pi_{A B}(\bar{s})}{\Pi_{B B}(\bar{s})}\right] V_{A, f}^{\mu}(\bar{s})
$$

which is the generalization of Eq. (34). Differentiating Eq. (28) with respect to $s$, setting $s=\bar{s}$, and using Eq. (39), we find

$$
\frac{d}{d \xi_{l}} \frac{V_{A, f}^{\mu}(\bar{s})}{\sqrt{\left[D(s) / \Pi_{B B}(s)\right]_{s=\bar{s}}^{\prime}}}=0
$$

for any choice of $f$. Noting that $\left[D(s) / \Pi_{B B}(s)\right]_{s=\bar{s}}^{\prime}=1-A^{\prime}(\bar{s})$ [cf. Eqs. (26) and (27)], we see that Eq. (40) generalizes Eq. (35) and implies the gauge independence of the pole residues in the mixed case.

The second term on the r.h.s. of Eq. (38) informs us that, in the case of mixing, loop corrections generate $O\left(g^{3}\right)$ gauge-dependent contributions to $V_{A, f}^{\mu}(s)$ that are not proportional to the lowest-order matrix elements $V_{A, f}^{\mu(0)}(s)$. (Here and in the following, a superfix $(n)$ indicates that the respective quantity is considered at $n$ loops.) They are proportional to the inverse propagator $D(s) / \Pi_{B B}(s)=\Pi_{A A}(s)-\Pi_{A B}^{2}(s) / \Pi_{B B}(s)$ and, therefore, vanish at $s=\bar{s}$. This is necessary, since they cannot be cancelled by other $O\left(g^{3}\right)$ contributions in the amplitude $V_{A, f}^{\mu}(\bar{s}) / \sqrt{\left[D(s) / \Pi_{B B}(s)\right]_{s=\bar{s}}^{\prime}}$. On the other hand, the proportionality to the inverse propagator implies that they contribute to the non-resonant amplitude, where they cancel other gauge-dependent terms. Well-known examples are contributions of this type to the neutral-current amplitudes of the SM involving the $Z-\gamma$ self-energy $A_{Z \gamma}(s)$, which cancel gauge-dependent terms in the photon-mediated amplitude and box diagrams [16]. We will refer to these terms as off-diagonal contributions.

The above-mentioned property of the residues has motivated a gauge-independent definition of partial width, to wit 8

$$
m_{2} \hat{\Gamma}_{f}=-\frac{1}{6} \sum_{\text {spins }} \int d \Phi_{f} \frac{Q_{\mu \nu} V_{f}^{\mu *}(\bar{s}) V_{f}^{\nu}(\bar{s})}{\left|1-A^{\prime}(\bar{s})\right|}
$$

where the integration is over the phase space of the final-state particles, a factor of $1 / 3$ arises from the average over the initial-state polarization, and a factor of $1 / 2$ from the familiar relation between $m_{2} \hat{\Gamma}_{f}$ and the integrated, squared amplitude. In the mixing case, it is understood that $V_{f}^{\mu}(s)$ is defined according to Eq. (37) and $A(s)$ according to Eq. (27). 
In Ref. [8], it was pointed out that $\sum_{f} \hat{\Gamma}_{f} \neq \Gamma_{2}$ when NNLO contributions are included, i.e. the sum of partial widths defined by means of Eq. (41) does not add up to the total width $\Gamma_{2}$ defined on the basis of the pole position $\bar{s}$ [Eq. (5)]. In principle, the lack of additivity can be circumvented by a simple rescaling: one defines the branching ratios by $B_{f}=\hat{\Gamma}_{f} / \sum_{f} \hat{\Gamma}_{f}$ and redefines the partial width as $\Gamma_{f}=B_{f} \Gamma_{2}$, so that $\sum_{f} \Gamma_{f}=\Gamma_{2}$ [8].

An analysis that leads to an alternative definition of partial widths, much closer to the conventional one, is based on the overall amplitude evaluated at $s=m_{2}^{2}$ [8],

$$
\mathcal{A}_{f i}\left(m_{2}^{2}\right)=-i \frac{Q_{\mu \nu} V_{f}^{\mu}\left(m_{2}^{2}\right) V_{i}^{\nu}\left(m_{2}^{2}\right)}{i m_{2} \Gamma_{2}-A\left(m_{2}^{2}\right)+A(\bar{s})}+\tilde{N}_{f i}
$$

where again $\tilde{N}_{f i}$ represents non-resonant contributions. It is understood that the gaugedependent, off-diagonal terms proportional to the inverse propagator $D\left(m_{2}^{2}\right) / \Pi_{B B}\left(m_{2}^{2}\right) \approx$ $i m_{2} \Gamma_{2}$, discussed in the paragraph after Eq. (40), are not included in $V_{f}^{\mu}\left(m_{2}^{2}\right)$ or $V_{i}^{\nu}\left(m_{2}^{2}\right)$, since they are needed to cancel other contributions of the same type in the non-resonant part $\tilde{N}_{f i}$.

Eq. (42) suggests the consideration of the amplitudes

$$
I_{f}\left(m_{2}^{2}\right)=\frac{1}{6} \sum_{\text {spins }} \int d \Phi_{f} Q_{\mu \nu} V_{f}^{\mu *}\left(m_{2}^{2}\right) V_{f}^{\nu}\left(m_{2}^{2}\right) .
$$

In fact, $-I_{f}\left(m_{2}^{2}\right) / m_{2}$ is the conventional expression for the partial width of the decay of the unstable particle into the physical state $f$, modulo its wave-function renormalization. In particular, $I_{f}\left(m_{2}^{2}\right)$ is the $f$-cut contribution to $I\left(m_{2}^{2}\right) \equiv \operatorname{Im} A\left(m_{2}^{2}\right)$ in the decomposition explained after Eq. (2), except that it is evaluated at the gauge-independent pole mass $m_{2}$, rather than the on-shell mass $M$. As explained in Ref. [8], if the unstable particle were an asymptotic state, the unitarity of the $S$ matrix would imply that $I\left(m_{2}^{2}\right)=F\left(m_{2}^{2}\right)$, where

$$
F\left(m_{2}^{2}\right) \equiv \sum_{f} I_{f}\left(m_{2}^{2}\right)
$$

Since the unstable particle is not an asymptotic state, this is not the case beyond NLO, and we expect a relation of the form

$$
I\left(m_{2}^{2}\right)=F\left(m_{2}^{2}\right)+G\left(m_{2}^{2}\right)
$$

where $G\left(m_{2}^{2}\right) \equiv I\left(m_{2}^{2}\right)-F\left(m_{2}^{2}\right)$ involves contributions from unphysical intermediate states, including would-be Goldstone bosons, Faddeev-Popov ghosts, or longitudinal modes of gauge bosons. By studying the gauge-independent difference $m_{2} \sum_{f} \hat{\Gamma}_{f}-m_{2} \Gamma_{2}$, it was shown in Ref. [8] that $G\left(m_{2}^{2}\right)$ is non-vanishing in $O\left(g^{6}\right)$, i.e. in NNLO.

A definition of branching ratios, very similar to the conventional one, was discussed in Ref. [8], to wit

$$
\tilde{B}_{f}=\frac{I_{f}\left(m_{2}^{2}\right)}{F\left(m_{2}^{2}\right)}
$$


It is manifestly additive: $\sum_{f} \tilde{B}_{f}=1$. The partial widths are then defined by

$$
\tilde{\Gamma}_{f}=\tilde{B}_{f} \Gamma_{2}
$$

In order to examine the gauge dependence of $I_{f}\left(m_{2}^{2}\right)$, we employ the NI of Eq. (32), with $s=m_{2}^{2}$, and find

$$
\begin{aligned}
\frac{\partial}{\partial \xi_{l}} I_{f}\left(m_{2}^{2}\right)= & 2 \operatorname{Re} \Lambda_{l}\left(m_{2}^{2}\right) I_{f}\left(m_{2}^{2}\right)+\frac{1}{6} \sum_{\text {spins }} \int d \Phi_{f} Q_{\mu \nu}\left[V_{f}^{\mu *}\left(m_{2}^{2}\right) \Pi\left(m_{2}^{2}\right) \Delta_{l, f}^{\nu}\left(m_{2}^{2}\right)\right. \\
& \left.+\Delta_{l, f}^{\mu *}\left(m_{2}^{2}\right) \Pi^{*}\left(m_{2}^{2}\right) V_{f}^{\nu}\left(m_{2}^{2}\right)\right] .
\end{aligned}
$$

In leading order, we have $\Pi\left(m_{2}^{2}\right)=-i I\left(m_{2}^{2}\right)$ and $\Delta_{l, f}^{\nu}\left(m_{2}^{2}\right)=\delta_{l}\left(m_{2}^{2}\right) V_{f}^{\nu}\left(m_{2}^{2}\right)$, where $\delta_{l}\left(m_{2}^{2}\right)=O\left(g^{2}\right)$ is independent of the final state $f$. An illustration of this property is provided by Eq. (56) and by the coefficient of $\Gamma_{Z, f}^{\mu(0)}(s)$ on the r.h.s. of Eq. (A.7). Thus, in leading order, Eq. (48) becomes

$$
\frac{\partial}{\partial \xi_{l}} I_{f}\left(m_{2}^{2}\right)=2\left[\operatorname{Re} \Lambda_{l}\left(m_{2}^{2}\right)+I\left(m_{2}^{2}\right) \operatorname{Im} \delta_{l}\left(m_{2}^{2}\right)\right] I_{f}\left(m_{2}^{2}\right)+O\left(g^{8}\right) .
$$

We note that $\Lambda_{l}\left(m_{2}^{2}\right), I\left(m_{2}^{2}\right), \delta_{l}\left(m_{2}^{2}\right)$, and $I_{f}\left(m_{2}^{2}\right)$ are of $O\left(g^{2}\right)$. Eq. (49) tells us that: (i) the gauge dependence of $I_{f}\left(m_{2}^{2}\right)$ starts in $O\left(g^{4}\right)$ (NLO), a well-known fact; and (ii) through $O\left(g^{6}\right)(\mathrm{NNLO}), \partial I_{f}\left(m_{2}^{2}\right) / \partial \xi_{l}$ is proportional to $I_{f}\left(m_{2}^{2}\right)$, with an $f$-independent coefficient. In turn, this proportionality implies the gauge independence of $\tilde{B}_{f}$ [Eq. (46)] through NNLO! The same conclusion holds in the presence of mixing, provided that, as explained after Eq. (42), gauge-dependent, off-diagonal terms proportional to $\mathrm{im}_{2} \mathrm{\Gamma}_{2}$ are not included in $V_{f}^{\mu}\left(m_{2}^{2}\right)$.

This observation explains a significant result of Ref. [8], namely it was shown in that work that, when the cross section of $e^{+} e^{-}$annihilation at the $Z^{0}$-boson peak in the SM is expressed in terms of the partial widths $\tilde{\Gamma}_{f}$, it is gauge independent through NNLO. From this conclusion it was inferred in Ref. [8] that the partial widths $\tilde{\Gamma}_{f}$ are also gauge independent through NNLO, i.e. through $O\left(g^{6}\right)$. We now see that this result is related to the proportionality between $\partial I_{f}\left(m_{2}^{2}\right) / \partial \xi_{l}$ and $I_{f}\left(m_{2}^{2}\right)$ with an $f$-independent coefficient, exhibited in Eq. (49) through $O\left(g^{6}\right)$. On the other hand, there is no reason to expect that such a proportionality survives in still higher orders.

Thus, although the definitions of Eqs. (46) and (47) provide gauge-independent results through NNLO, and this is sufficient for the phenomenological requirements of electroweak physics in the foreseeable future, they are not completely satisfactory from the theoretical point of view, since they are not expected to be gauge independent in still higher orders.

Next, we apply the NI to obtain information about the function $G\left(m_{2}^{2}\right)$ introduced in Eq. (45). Taking the imaginary part of Eq. (8), setting $s=m_{2}^{2}$, and recalling from Eq. (7) that $\operatorname{Im} \Pi\left(m_{2}^{2}\right)=-I\left(m_{2}^{2}\right)$, we have

$$
\frac{\partial}{\partial \xi_{l}} I\left(m_{2}^{2}\right)=2 \operatorname{Re} \Lambda_{l}\left(m_{2}^{2}\right) I\left(m_{2}^{2}\right)+2 \operatorname{Im} \Lambda_{l}\left(m_{2}^{2}\right) \operatorname{Re}\left[A\left(m_{2}^{2}\right)-A(\bar{s})\right] .
$$


Through $O\left(g^{6}\right)$, this becomes

$$
\frac{\partial}{\partial \xi_{l}} I\left(m_{2}^{2}\right)=2 \operatorname{Re} \Lambda_{l}\left(m_{2}^{2}\right) I\left(m_{2}^{2}\right)-2 m_{2} \Gamma_{2} \operatorname{Im} \Lambda_{l}\left(m_{2}^{2}\right) I^{\prime}\left(m_{2}^{2}\right)+O\left(g^{8}\right) .
$$

Inserting Eq. (45) into Eq. (51) and subtracting

$$
\frac{\partial}{\partial \xi_{l}} F\left(m_{2}^{2}\right)=2\left[\operatorname{Re} \Lambda_{l}\left(m_{2}^{2}\right)+I\left(m_{2}^{2}\right) \operatorname{Im} \delta_{l}\left(m_{2}^{2}\right)\right] F\left(m_{2}^{2}\right),
$$

a relation that follows from Eq. (49), we find

$$
\frac{\partial}{\partial \xi_{l}} G\left(m_{2}^{2}\right)=\frac{m_{2} \Gamma_{2}}{2} \frac{\partial}{\partial \xi_{l}}\left[I^{\prime}\left(m_{2}^{2}\right)\right]^{2}-2 m_{2}^{2} \Gamma_{2}^{2} \operatorname{Im} \delta_{l}\left(m_{2}^{2}\right)+O\left(g^{8}\right) .
$$

In Eq. (53), we have neglected terms of $O\left(g^{8}\right)$ and employed

$$
\operatorname{Im} \Lambda_{l}\left(m_{2}^{2}\right)=-\frac{1}{2} \frac{\partial}{\partial \xi_{l}} I^{\prime}\left(m_{2}^{2}\right)+O\left(g^{4}\right),
$$

a result that follows from Eq. (8). Eq. (53) tells us that $G\left(m_{2}^{2}\right)$ differs from zero in $O\left(g^{6}\right)$ (NNLO), a conclusion also reached by a different argument in Ref. [8]. In fact, Eq. (16) of Ref. [8] presents an explicit expression for the $O\left(g^{6}\right)$ contribution to $G\left(m_{2}^{2}\right)$ in the $Z^{0}$-boson case of the SM, obtained by considering the difference between the two gauge-independent amplitudes $m_{2} \sum_{f} \hat{\Gamma}_{f}$ and $m_{2} \Gamma_{2}$. As shown in that expression, in the $Z^{0}$-boson case, the leading contribution to $G\left(m_{2}^{2}\right)$ depends on the gauge parameter $\xi_{W}$ associated with the $W$ boson. Differentiating Eq. (16) of Ref. [8] with respect to $\xi_{W}$, we find

$$
\frac{\partial}{\partial \xi_{W}} G\left(m_{2}^{2}\right)=\frac{m_{2} \Gamma_{2}}{2} \frac{\partial}{\partial \xi_{W}}\left[I^{\prime}\left(m_{2}^{2}\right)\right]^{2}-g^{2} c_{w}^{2} m_{2}^{2} \Gamma_{2}^{2} \frac{\partial}{\partial \xi_{W}}\left[\left(\xi_{W}-1\right) \operatorname{Im} \eta_{W}\left(m_{2}^{2}\right)\right]+O\left(g^{8}\right),
$$

where $c_{w} \equiv \cos \theta_{w}$, with $\theta_{w}$ being the weak mixing angle, and $\eta_{W}(s)$ is a gauge-dependent amplitude introduced in Ref. [16]. Comparison of Eqs. (53) and (55) shows that the two expressions have the same structure with the identification

$$
\operatorname{Im} \delta_{W}\left(m_{2}^{2}\right)=\frac{g^{2} c_{w}^{2}}{2} \frac{\partial}{\partial \xi_{W}}\left[\left(\xi_{W}-1\right) \operatorname{Im} \eta_{W}\left(m_{2}^{2}\right)\right] .
$$

Thus, the NI permit us to understand the non-vanishing of $G\left(m_{2}^{2}\right)$ and the structure of its leading contribution. As shown in Ref. [B] , the contribution to $G\left(m_{2}^{2}\right)$ involving $\operatorname{Im} \eta_{W}\left(m_{2}^{2}\right)$ plays a crucial role in ensuring the gauge independence of the peak cross section through NNLO. In fact, it cancels the gauge dependence of the interference between the resonant amplitude and the box diagrams! 


\section{EXTENSION TO ALL ORDERS OF THE GAUGE INDEPENDENCE OF EQS. (3.18) AND (3.19)}

A strategy to extend to all orders the gauge independence of Eq. (46), and therefore Eq. (47), involves a redefinition of the vertex parts $V_{f}^{\mu}\left(m_{2}^{2}\right)$. In general, $V_{f}^{\mu}(s)$ may be regarded as a function $V_{f}^{\mu}\left(s, m_{2}^{2}, m_{2} \Gamma_{2}\right)$. The explicit dependence on $m_{2} \Gamma_{2}$ arises from specific cut contributions, or, equivalently, from terms involving $\operatorname{Im} A(s)$. As an illustration, we may consider two-loop contributions to the $Z$ - $\gamma$ self-energy $A_{Z \gamma}(s)$ involving virtual fermion-antifermion pairs with a vertex correction attached to the photon ending. A cut across the fermion-antifermion pairs, summed over all pairs, leads at $s=m_{2}^{2}$ to a contribution proportional to $i m_{2} \Gamma_{2}$. The fact that higher-order corrections generate contributions involving $m_{2} \Gamma_{2}$ may also be inferred more generally by examining the structure of Eqs. (28) and (38). We note that the terms involving $D(s) / \Pi_{B B}(s)$ in these equations are proportional to $s-\bar{s}=s-m_{2}^{2}+i m_{2} \Gamma_{2}$, and it is clear that $i m_{2} \Gamma_{2}$ must be induced by contributions of higher order than those leading to $s-m_{2}^{2}$. In the notation of this section, the amplitudes $V_{f}^{\mu}(\bar{s})$ and $V_{f}^{\mu}\left(m_{2}^{2}\right)$ are identified with $V_{f}^{\mu}(\bar{s})=V_{f}^{\mu}\left(\bar{s}, m_{2}^{2}, m_{2} \Gamma_{2}\right)$ and $V_{f}^{\mu}\left(m_{2}^{2}\right)=V_{f}^{\mu}\left(m_{2}^{2}, m_{2}^{2}, m_{2} \Gamma_{2}\right)$, respectively. A modification that renders Eqs. (46) and (47) gauge independent to all orders involves replacing $V_{f}^{\mu}\left(m_{2}^{2}\right)$ in Eq. (43) by $V_{f}^{\mu}\left(m_{2}^{2}\right) \equiv \lim _{\Gamma_{2} \rightarrow 0} V_{f}^{\mu}\left(\bar{s}, m_{2}^{2}, m_{2} \Gamma_{2}\right)=V_{f}^{\mu}\left(m_{2}^{2}, m_{2}^{2}, 0\right)$. We emphasize that $\hat{V}_{f}^{\mu}\left(m_{2}^{2}\right)$ differs from the usual definition of $V_{f}^{\mu}\left(m_{2}^{2}\right)$. Setting $s=m_{2}^{2}$ in Eq. (38) and taking the limit $\Gamma_{2} \rightarrow 0$, we see that

$$
\frac{\partial}{\partial \xi_{l}} \hat{V}_{A, f}^{\mu}\left(m_{2}^{2}\right)=\left[\Lambda_{l, A}^{A}\left(m_{2}^{2}\right)-\frac{\Lambda_{l, B}^{A}\left(m_{2}^{2}\right) \Pi_{A B}\left(m_{2}^{2}\right)}{\Pi_{B B}\left(m_{2}^{2}\right)}\right]_{\Gamma_{2}=0} \hat{V}_{A, f}^{\mu}\left(m_{2}^{2}\right) .
$$

The crucial point is that, in the limit $\Gamma_{2} \rightarrow 0$, the second term on the r.h.s. of Eq. (38) vanishes. This has the effect of excluding from $\hat{V}_{A, f}^{\mu}\left(m_{2}^{2}\right)$ all the gauge-dependent (diagonal as well as off-diagonal) contributions that are proportional to $m_{2} \Gamma_{2}$. The physical meaning of this exclusion is discussed later.

Replacing $I_{f}\left(m_{2}^{2}\right)$ in Eq. (43) by

$$
\hat{I}_{f}\left(m_{2}^{2}\right)=\frac{1}{6} \sum_{\text {spins }} \int d \Phi_{f} Q_{\mu \nu} \hat{V}_{f}^{\mu *}\left(m_{2}^{2}\right) \hat{V}_{f}^{\nu}\left(m_{2}^{2}\right),
$$

Eq. (57) leads to the proportionality between $\partial \hat{I}_{f}\left(m_{2}^{2}\right) / \partial \xi_{l}$ and $\hat{I}_{f}\left(m_{2}^{2}\right)$ with an $f$-independent coefficient. In turn, this implies the gauge independence to all orders of the modified definitions

$$
\begin{gathered}
\hat{B}_{f}=\frac{\hat{I}_{f}\left(m_{2}^{2}\right)}{\hat{F}\left(m_{2}^{2}\right)}, \\
\hat{\Gamma}_{f}=\hat{B}_{f} \Gamma_{2},
\end{gathered}
$$

where $\hat{F}\left(m_{2}^{2}\right) \equiv \sum_{f} \hat{I}_{f}\left(m_{2}^{2}\right)$. 
A corresponding gauge-independent definition of residues is obtained by considering

$$
\lim _{\Gamma_{2} \rightarrow 0} \frac{Q_{\mu \nu} V_{f}^{\mu}(\bar{s}) V_{f}^{\nu}(\bar{s})}{1-A^{\prime}(\bar{s})}=\frac{Q_{\mu \nu} \hat{V}_{f}^{\mu}\left(m_{2}^{2}\right) \hat{V}_{f}^{\nu}\left(m_{2}^{2}\right)}{\left[1-A^{\prime}(\bar{s})\right]_{\Gamma_{2}=0}} .
$$

The gauge independence of Eq. (60) follows by taking the limit $\Gamma_{2} \rightarrow 0$ of Eq. (35).

In terms of Eq. (60), the overall amplitude of Eq. (31) may be expressed as

$$
\mathcal{A}_{f i}(s)=-i \frac{Q_{\mu \nu} \hat{V}_{f}^{\mu}\left(m_{2}^{2}\right) \hat{V}_{i}^{\nu}\left(m_{2}^{2}\right)}{(s-\bar{s})\left[1-A^{\prime}(\bar{s})\right]_{\Gamma_{2}=0}}+\hat{N}_{f i} .
$$

The physical meaning of the limit $\Gamma_{2} \rightarrow 0$ can be easily understood by comparing the first terms in Eqs. (31) and (61). The residues of $1 /(s-\bar{s})$ in the two expressions differ by terms of $O\left(g^{4} \Gamma_{2} / m_{2}\right)$ (NNLO). Such terms give contributions of zeroth order in $\Gamma_{2}$ to the peak amplitude $\left(s=m_{2}^{2}\right)$ and, therefore, may be regarded as non-resonant. It should be stressed that, in this approach, terms of $O\left(\left(\Gamma_{2} / m_{2}\right)^{n}\right)$, where $n \geq 1$, are not neglected, but they are rather incorporated in the non-resonant amplitude. Thus, the two expressions differ, in a gauge-independent manner, in the precise identification of resonant and nonresonant contributions. Although the formulation of Eq. (31) is probably more elegant, that of Eq. (61) is closer to the calculations carried out by most particle physicists.

\section{$5 \quad$ NI FOR BOX FUNCTIONS}

The gauge independence of the complete amplitude can be tested by considering the NI for the box functions $B_{f i}(s)$. In the absence of mixing, we have

$$
\frac{\partial}{\partial \xi_{l}} B_{f i}(s)=i Q_{\mu \nu}\left[\Delta_{l, f}^{\mu}(s) V_{i}^{\nu}(s)+V_{f}^{\mu}(s) \Delta_{l, i}^{\nu}(s)\right]
$$

(see, e.g., Eq. (46) of Ref. [5] and Eq. (14) of Ref. [13]). Eq. (62) only involves the functions $\Delta_{l, f}^{\mu}(s)$ appearing in the NI for vertex functions [Eq. [32)]. This is due to BRST symmetry and the fact that the external states $i$ and $f$ are on shell. Indeed, in the NI for off-shell Green functions, there are additional contributions proportional to the field equations .

Knowing the gauge dependence of all essential building blocks, we can find the most general combination of self-energy, vertex, and box contributions that is gauge independent for any value of $s$. From the NI for self-energies [Eq. (8)], we can compute the function $\Lambda_{l}(s)$ as

$$
\Lambda_{l}(s)=\frac{1}{2 \Pi(s)} \frac{\partial}{\partial \xi_{l}} \Pi(s) .
$$

Inserting $\Lambda_{l}(s)$ into the NI for vertices [Eq. (32)] and solving for $\Delta_{l, f}^{\mu}(s)$, we find

$$
\Delta_{l, f}^{\mu}(s)=\frac{1}{\sqrt{\Pi(s)}} \frac{\partial}{\partial \xi_{l}} \frac{V_{f}^{\mu}(s)}{\sqrt{\Pi(s)}},
$$


and analogously for $\Delta_{l, i}^{\nu}(s)$. Combining Eqs. (62) and (64), the variation of $B_{f i}(s)$ with respect to the gauge parameter becomes

$$
\frac{\partial}{\partial \xi_{l}} B_{f i}(s)=i \frac{\partial}{\partial \xi_{l}} \frac{Q_{\mu \nu} V_{f}^{\mu}(s) V_{i}^{\nu}(s)}{\Pi(s)} .
$$

Eq. (65) implies that the most general gauge-independent combination is an arbitrary function of

$$
\Phi_{f i}(s)=-i \frac{Q_{\mu \nu} V_{f}^{\mu}(s) V_{i}^{\nu}(s)}{\Pi(s)}+B_{f i}(s) .
$$

Finally, requiring that the combination has a simple pole given by the zero of $\Pi(s)$, we see that it must be a linear function of $\Phi_{f i}(s)$. Thus, the NI tell us that, subject to the latter requirement, the most general combination that is gauge independent for arbitrary $s$ is the physical amplitude! This result can be readily extended to the case of field mixing.

It is interesting to observe that the argument presented in this section may be reversed to give a simple derivation of the functional structure of Eqs. (32) and (62). Invoking the gauge independence of the $S$-matrix element given in Eq. (66) and noting that $\partial B_{f i}(s) / \partial \xi_{l}$ does not contain terms proportional to $1 / \Pi(s)$, one concludes that the same is true of $-i \partial\left[Q_{\mu \nu} V_{f}^{\mu}(s) V_{i}^{\nu}(s) / \Pi(s)\right] / \partial \xi_{l}$. Observing that $V_{f}^{\mu}(s)$ and $V_{i}^{\nu}(s)$ are independent functions and employing Eq. (8), one readily finds that $\partial V_{f}^{\mu}(s) / \partial \xi_{l}$ satisfies a relation with the functional structure of Eq. (32), subject to the constraint that the function $\Delta_{l, f}^{\mu}(s)$ does not contain 1/ח(s) contributions. Differentiating Eq. (66) with respect to $\xi_{l}$, inserting Eqs. (8) and (32), and invoking once more the gauge independence of the $S$ matrix, one obtains Eq. (62).

\section{ANALYTICAL PROPERTIES AND NI}

As is well known, the analytical properties of the Green function $\Pi(s)$ permit the use of Cauchy's theorem,

$$
\Pi(s)=\frac{1}{2 \pi i} \oint_{\gamma} d s^{\prime} \frac{\Pi\left(s^{\prime}\right)}{s^{\prime}-s},
$$

where $\gamma$ is a closed contour in the complex $s^{\prime}$ plane that encircles the point $s$ counterclockwise. Eq. (67) and the distribution-based relation

$$
\lim _{\epsilon \rightarrow 0} \frac{1}{s-s^{\prime}+i \epsilon}=P \frac{1}{s-s^{\prime}}-i \pi \delta\left(s-s^{\prime}\right)
$$

where $P$ denotes the principal value, leads to the derivation of dispersion relations and sum rules.

Using Eq. (67) and the NI of Eq. (8), we have

$$
\frac{\partial}{\partial \xi_{l}} \Pi(s)=\frac{1}{2 \pi i} \oint_{\gamma} d s^{\prime} \frac{2 \Lambda_{l}\left(s^{\prime}\right) \Pi\left(s^{\prime}\right)}{s^{\prime}-s}=\frac{1}{(2 \pi i)^{2}} \oint_{\gamma} d s^{\prime} \oint_{\gamma^{\prime}} d s^{\prime \prime} \frac{2 \Lambda_{l}\left(s^{\prime}\right) \Pi\left(s^{\prime \prime}\right)}{\left(s^{\prime}-s\right)\left(s^{\prime \prime}-s^{\prime}\right)}
$$


where the contour $\gamma^{\prime}$ encircles $\gamma$ counterclockwise. Rewriting the factor $1 /\left[\left(s^{\prime}-s\right)\left(s^{\prime \prime}-s^{\prime}\right)\right]$, the last member of Eq. (69) becomes

$$
\frac{\partial}{\partial \xi_{l}} \Pi(s)=\frac{1}{(2 \pi i)^{2}} \oint_{\gamma} d s^{\prime} \oint_{\gamma^{\prime}} d s^{\prime \prime} 2 \Lambda_{l}\left(s^{\prime}\right) \Pi\left(s^{\prime \prime}\right)\left[\frac{1}{\left(s^{\prime}-s\right)\left(s^{\prime \prime}-s\right)}+\frac{1}{\left(s^{\prime \prime}-s^{\prime}\right)\left(s^{\prime \prime}-s\right)}\right],
$$

where the second term vanishes, since the integration over $s^{\prime}$ along the contour $\gamma$ does not encircle any singularity. Therefore, we find

$$
\frac{\partial}{\partial \xi_{l}} \Pi(s)=\left[\frac{1}{2 \pi i} \oint_{\gamma} d s^{\prime} \frac{2 \Lambda_{l}\left(s^{\prime}\right)}{s^{\prime}-s}\right] \Pi(s),
$$

which implies that $\Lambda_{l}(s)$ admits a spectral representation, analogous to Eq. (67), that is compatible with the analyticity of Green functions.

A clear example of the analyticity of the functions $\Lambda_{l}(s)$ can be read off from the absorptive part of the Higgs-boson two-point function $\operatorname{Im} \Pi_{H H}(s)$ presented in Ref. [4]. From the imaginary part of Eq. (8) at one loop, we have

$$
\frac{\partial}{\partial \xi_{W}} \operatorname{Im} \Pi_{H H}^{(1)}(s)=2\left(s-M_{H}^{2}\right) \operatorname{Im} \Lambda_{W, H}^{H(1)}(s) .
$$

Comparison with Ref. [四] shows that

$$
\begin{aligned}
\operatorname{Im} \Lambda_{W, H}^{H(1)}(s)= & \frac{G M_{W}^{2}}{2}\left(s+M_{H}^{2}\right)\left[\frac{1}{2 s}\left(1-\frac{4 \xi_{W} M_{W}^{2}}{s}\right)^{-1 / 2} \theta\left(s-4 \xi_{W} M_{W}^{2}\right)\right. \\
& \left.-\left(1-\frac{4 \xi_{W} M_{W}^{2}}{s}\right)^{1 / 2} \delta\left(s-4 \xi_{W} M_{W}^{2}\right)\right],
\end{aligned}
$$

where $G=G_{\mu} /(2 \pi \sqrt{2})$, with $G_{\mu}$ being the muon decay constant. Although the second term seems to violate the analyticity of Green functions because of the presence the $\delta$ function, the factor $\left(1-4 \xi_{W} M_{W}^{2} / s\right)^{1 / 2}$ leads to the vanishing of this contribution. Thus, we obtain

$$
\operatorname{Im} \Lambda_{W, H}^{H(1)}(s)=\frac{G M_{W}^{2}}{4}\left(1+\frac{M_{H}^{2}}{s}\right)\left(1-\frac{4 \xi_{W} M_{W}^{2}}{s}\right)^{-1 / 2} \theta\left(s-4 \xi_{W} M_{W}^{2}\right) .
$$

The function $\Lambda_{W, H}^{H(1)}(s)$ can also be obtained by a direct evaluation of the relevant Feynman diagrams. In fact, Eq. (74) has the structure one expects from such a computation. It shows that the one-loop Green function $\Lambda_{W, H}^{H(1)}(s)$ contains absorptive parts, but they are proportional to $\theta$ functions centered at some unphysical thresholds. This is due to the fact that, in the diagrams for $\Lambda_{W, H}^{H(1)}(s)$, only unphysical modes propagate at one loop. However, $\theta$ functions centered at physical thresholds may appear at higher orders.

\footnotetext{
${ }^{1}$ The decoupling of unphysical modes, the mixing with the neutral would-be Goldstone boson, and the NI for Higgs bosons are discussed in Ref. [5].
} 
The NI tell us that the gauge dependence of a Green function is described by another Green function, which can be computed in terms of Feynman rules. The factorization implied by the NI is far from being trivial, and the Higgs-boson example shows how analyticity works in the factorization of the second member of the NI.

\section{CONCLUSIONS}

In this paper, we examined fundamental properties of unstable particles, such as their masses, widths, and partial widths, in the light of the NI, which describe the gauge dependence of Green functions.

In Sec. II, we applied the NI to show that the conventional definitions of mass and width of unstable particles are gauge dependent in NNLO. This shows that, in the gaugetheory context, the conventional treatment of unstable particles is strictly valid through NLO. For completeness, in the same section, we revisited the formal proof, to all orders, of the gauge independence of the pole position $\bar{s}$ [5].

In Sec. III, we applied the NI for vertex functions to prove the gauge independence of the pole residues. This motivates a gauge-independent definition of partial widths. As explained in Ref. [8], this definition does not satisfy the additivity property in NNLO. However, this problem can be circumvented by a judicious rescaling of the partial widths.

We then considered an alternative definition of branching ratios $\tilde{B}_{f}$ and partial widths $\tilde{\Gamma}_{f}$ that are manifestly additive and closely resemble the conventional ones. Using the NI, we showed that $\tilde{B}_{f}$ and $\tilde{\Gamma}_{f}$ are gauge independent through NNLO. This explains a significant result obtained in Ref. [8], namely that the cross section of $e^{+} e^{-}$annihilation at the $Z^{0}$-boson peak in the SM, expressed in terms of $\tilde{\Gamma}_{f}$, is gauge independent through NNLO. Although this result is sufficient for the phenomenological requirements of electroweak physics in the foreseeable future, $\tilde{B}_{f}$ and $\tilde{\Gamma}_{f}$ are not expected to be gauge independent in still higher orders.

We also used the NI to show that the usual assumption that $\operatorname{Im} A\left(m_{2}^{2}\right)$ can be expressed as a sum of physical cut contributions fails in NNLO. The difference between these two quantities is given by a function $G\left(m_{2}^{2}\right)$ that emerges in NNLO. In Ref. [8], $G\left(m_{2}^{2}\right)$ was shown to be non-vanishing by studying the difference between two gauge-independent definitions of total width based, respectively, on the pole residues and the pole position. In the present paper, we employed the NI to derive an expression for $G\left(m_{2}^{2}\right)$ with the same mathematical structure.

In Sec. IV, we showed how to modify the alternative definition of branching ratios, discussed in Sec. III, in order to extend the gauge independence to all orders.

In Sec. V, we discussed the NI for box diagrams and showed that the physical amplitude is the most general combination of self-energy, vertex, and box contributions that is gauge independent for arbitrary $s$ ! Reversing the argument, we also showed that the functional structure of the NI for vertex and box functions can be derived starting from the gauge independence of the $S$ matrix and well-known properties of the box amplitudes. It should also be emphasized that the gauge independence of the physical amplitude implies the 
same property for the coefficients of its Laurent expansion.

Section VI discusses the analytic properties of the Green functions $\Lambda_{l}(s)$ that play an important role in the NI.

The Appendix gives the explicit one-loop relations between the functions $\Lambda_{l, \alpha}^{\delta}(s)$ and $\Delta_{l, f}^{\delta, \mu}(s)$ that occur in the NI and the calculations of Ref. [16] in the $Z$ - $\gamma$ sector of the SM.

\section{Acknowledgements}

B.A.K. and A.S. thank the members of the Physics Department of the University of Barcelona and the Theoretical Physics Division of CERN, respectively, for their kind hospitality during September 2001. The research of P.A.G. and A.S. was supported in part by National Science Foundation through Grant No. PHY-0070787. The research of B.A.K. was supported in part by the Deutsche Forschungsgemeinschaft through Grant No. KN 365/1-1, by the Bundesministerium für Bildung und Forschung through Grant No. 05 HT1GUA/4, and by the European Commission through the Research Training Network Quantum Chromodynamics and the Deep Structure of Elementary Particles.

\section{A ONE-LOOP CONTRIBUTIONS TO NI FUNC- TIONS}

In this Appendix, we present the one-loop contributions to the NI for the $Z-\gamma$ sector of the SM. In particular, we discuss the relation between the NI Green functions $\Lambda_{l, \alpha}^{\delta}(s)$ and $\Delta_{l, f}^{\delta, \mu}(s)$ defined in Eqs. (22) and (36), respectively, and the one-loop computations of Ref. [16].

According to Eq. (22), in the $Z-\gamma$ case, the transverse self-energies $\Pi_{Z Z}(s), \Pi_{Z \gamma}(s)$, and $\Pi_{\gamma \gamma}(s)$ satisfy

$$
\begin{aligned}
\frac{\partial}{\partial \xi_{l}} \Pi_{Z Z}(s) & =2 \sum_{\delta=Z, \gamma} \Lambda_{l, Z}^{\delta}(s) \Pi_{\delta Z}(s), \\
\frac{\partial}{\partial \xi_{l}} \Pi_{Z \gamma}(s) & =\sum_{\delta=Z, \gamma}\left[\Lambda_{l, Z}^{\delta}(s) \Pi_{\delta \gamma}(s)+\Lambda_{l, \gamma}^{\delta}(s) \Pi_{\delta Z}(s)\right], \\
\frac{\partial}{\partial \xi_{l}} \Pi_{\gamma \gamma}(s) & =2 \sum_{\delta=Z, \gamma} \Lambda_{l, \gamma}^{\delta}(s) \Pi_{\delta \gamma}(s),
\end{aligned}
$$

where $\Lambda_{l, Z}^{Z}(s), \Lambda_{l, Z}^{\gamma}(s), \Lambda_{l, \gamma}^{Z}(s)$, and $\Lambda_{l, \gamma}^{\gamma}(s)$ involve the gauge fields $Z$ or $\gamma$ (lower index), the BRST transformation of the gauge parameter $\xi_{l}$, and the sources of the BRST variations associated with $Z$ or $\gamma$ (upper index). As implied by the statement before Eq. (23), the complex pole positions $\bar{s}$ are given by the equation

$$
\Pi_{Z Z}(\bar{s}) \Pi_{\gamma \gamma}(\bar{s})-\Pi_{Z \gamma}^{2}(\bar{s})=0
$$


which, being a quadratic polynomial in $\bar{s}$ at tree level, has two solutions at all orders. Actually, one solution is trivially $\bar{s}=0$, due to BRST symmetry (cf. Ref. [17]).

In order to compare this with the explicit one-loop computations of Ref. [16], we reduce Eq. (A.1) to the one-loop level using the fact that the $\Lambda_{l, \alpha}^{\delta}(s)$ and $\Pi_{Z \gamma}(s)$ functions are of $O\left(g^{2}\right)$ :

$$
\begin{aligned}
\frac{\partial}{\partial \xi_{l}} \Pi_{Z Z}^{(1)}(s) & =2\left(s-M_{Z}^{2}\right) \Lambda_{l, Z}^{Z(1)}(s), \\
\frac{\partial}{\partial \xi_{l}} \Pi_{Z \gamma}^{(1)}(s) & =\left[s \Lambda_{l, Z}^{\gamma(1)}(s)+\left(s-M_{Z}^{2}\right) \Lambda_{l, \gamma}^{Z(1)}(s)\right], \\
\frac{\partial}{\partial \xi_{l}} \Pi_{\gamma \gamma}^{(1)}(s) & =2 s \Lambda_{l, \gamma}^{\gamma(1)}(s) .
\end{aligned}
$$

Comparing Eq. (ब.3) with the results of Ref. [16] and employing the functions $v_{W}(s)$ and $\eta_{W}(s)$ defined in that work, we obtain

$$
\begin{aligned}
& \int_{1}^{\xi_{W}} d \xi_{W}^{\prime} \Lambda_{W, Z}^{Z(1)}\left(s, \xi_{W}^{\prime}\right)=-g^{2} c_{w}^{2}\left(\xi_{W}-1\right)\left[v_{W}(s)+\frac{1}{2}\left(s-M_{Z}^{2}\right) \eta_{W}(s)\right] \\
& \int_{1}^{\xi_{W}} d \xi_{W}^{\prime} \Lambda_{W, Z}^{\gamma(1)}\left(s, \xi_{W}^{\prime}\right)=-g^{2} s_{w} c_{w}\left(\xi_{W}-1\right)\left[v_{W}(s)+\frac{1}{2}\left(s-M_{Z}^{2}\right) \eta_{W}(s)\right], \\
& \int_{1}^{\xi_{W}} d \xi_{W}^{\prime} \Lambda_{W, \gamma}^{Z(1)}\left(s, \xi_{W}^{\prime}\right)=-g^{2} s_{w} c_{w}\left(\xi_{W}-1\right)\left[v_{W}(s)+\frac{s}{2} \eta_{W}(s)\right] \\
& \int_{1}^{\xi_{W}} d \xi_{W}^{\prime} \Lambda_{W, \gamma}^{\gamma(1)}\left(s, \xi_{W}^{\prime}\right)=-g^{2} s_{w}^{2}\left(\xi_{W}-1\right)\left[v_{W}(s)+\frac{s}{2} \eta_{W}(s)\right]
\end{aligned}
$$

where $s_{w} \equiv \sin \theta_{w}$. The other functions $\Lambda_{l, \alpha}^{\delta(1)}\left(s, \xi_{k}\right)$, with $l \neq W$ and $\alpha, \delta=Z, \gamma$, vanish. From Eq. (A.4), we can immediately see that there are only two functionally independent Green functions, viz

$$
\begin{aligned}
& \Lambda_{W, Z}^{3(1)}\left(s, \xi_{W}\right)=\Lambda_{W, Z}^{Z(1)}\left(s, \xi_{W}\right)+\frac{s_{w}}{c_{w}} \Lambda_{W, Z}^{\gamma(1)}\left(s, \xi_{W}\right), \\
& \Lambda_{W, \gamma}^{3(1)}\left(s, \xi_{W}\right)=\frac{c_{w}}{s_{w}} \Lambda_{W, \gamma}^{Z(1)}\left(s, \xi_{W}\right)+\Lambda_{W, \gamma}^{\gamma(1)}\left(s, \xi_{W}\right) .
\end{aligned}
$$

This result is expected on the basis of BRST symmetry. In fact, following Ref. [17], one only introduces the source coupled to non-linear BRST transformations; for example, one needs the source for the BRST variations of the gauge bosons $W_{\mu}^{i}$ of the $S U(2)$ triplet, but not for the abelian gauge field $B_{\mu}$. This implies that there are only two independent functions $\Lambda_{l, \alpha}^{\delta}\left(s, \xi_{k}\right)$ in the $Z-\gamma$ sector.

As for the vertex functions, from Eq. (36) at one loop, we have

$$
\begin{aligned}
& \frac{\partial}{\partial \xi_{l}} \Gamma_{Z, f}^{\mu(1)}(s)=\sum_{\delta=Z, \gamma} \Lambda_{l, Z}^{\delta(1)}(s) \Gamma_{\delta, f}^{\mu(0)}(s)+\left(s-M_{Z}^{2}\right) \Delta_{l, f}^{Z, \mu(1)}(s), \\
& \frac{\partial}{\partial \xi_{l}} \Gamma_{\gamma, f}^{\mu(1)}(s)=\sum_{\delta=Z, \gamma} \Lambda_{l, \gamma}^{\delta(1)}(s) \Gamma_{\delta, f}^{\mu(0)}(s)+s \Delta_{l, f}^{\gamma, \mu(1)}(s) .
\end{aligned}
$$


Comparing with the results of Ref. [16], we find

$$
\begin{aligned}
& \int_{1}^{\xi_{W}} d \xi_{W}^{\prime} \Delta_{W, f}^{Z, \mu(1)}\left(s, \xi_{W}^{\prime}\right)=\frac{g^{2} c_{w}^{2}}{2}\left(\xi_{W}-1\right) \eta_{W}(s)\left[\Gamma_{Z, f}^{\mu(0)}(s)+\frac{s_{w}}{c_{w}} \Gamma_{\gamma, f}^{\mu(0)}(s)\right], \\
& \int_{1}^{\xi_{W}} d \xi_{W}^{\prime} \Delta_{W, f}^{\gamma, \mu(1)}\left(s, \xi_{W}^{\prime}\right)=\frac{g^{2} s_{w} c_{w}}{2}\left(\xi_{W}-1\right) \eta_{W}(s)\left[\Gamma_{Z, f}^{\mu(0)}(s)+\frac{s_{w}}{c_{w}} \Gamma_{\gamma, f}^{\mu(0)}(s)\right] .
\end{aligned}
$$

The other functions $\Delta_{l, f}^{\delta, \mu(1)}\left(s, \xi_{k}\right)$, with $l \neq W$ and $\delta=Z, \gamma$, vanish. We note again that $\Delta_{W, f}^{\delta, \mu(1)}\left(s, \xi_{W}\right)$, with $\delta=Z, \gamma$, are not independent, but proportional to

$$
\Delta_{W, f}^{3, \mu(1)}\left(s, \xi_{W}\right)=\Delta_{W, f}^{Z, \mu(1)}\left(s, \xi_{W}\right)+\frac{s_{w}}{c_{w}} \Delta_{W, f}^{\gamma, \mu(1)}\left(s, \xi_{W}\right),
$$

which is expected from BRST symmetry, as explained above. Moreover, we see that they are proportional to a combination of tree-level vertex functions. By inserting Eqs. (A.4) and (A.7) in Eq. (A.6), we obtain

$$
\begin{aligned}
& \frac{\partial}{\partial \xi_{W}} \Gamma_{Z, f}^{\mu(1)}\left(s, \xi_{W}\right)=-g^{2} c_{w}^{2} \frac{\partial}{\partial \xi_{W}}\left[\left(\xi_{W}-1\right) v_{W}(s)\right]\left[\Gamma_{Z, f}^{\mu(0)}(s)+\frac{s_{w}}{c_{w}} \Gamma_{\gamma, f}^{\mu(0)}(s)\right], \\
& \frac{\partial}{\partial \xi_{W}} \Gamma_{\gamma, f}^{\mu(1)}\left(s, \xi_{W}\right)=-g^{2} s_{w} c_{w} \frac{\partial}{\partial \xi_{W}}\left[\left(\xi_{W}-1\right) v_{W}(s)\right]\left[\Gamma_{Z, f}^{\mu(0)}(s)+\frac{s_{w}}{c_{w}} \Gamma_{\gamma, f}^{\mu(0)}(s)\right],
\end{aligned}
$$

in agreement with the vertex computations in Ref. [16].

\section{References}

[1] A. Sirlin, Phys. Rev. Lett. 67, 2127 (1991); Phys. Lett. B 267, 240 (1991).

[2] M. Consoli and A. Sirlin, in Physics at LEP, CERN Yellow Report No. 86-02, 1986, Vol. 1, p. 63; S. Willenbrock and G. Valencia, Phys. Lett. B 259, 373 (1991); R. G. Stuart, Phys. Lett. B 262, 113 (1991); 272, 353 (1991); Phys. Rev. Lett. 70, 3193 (1993); Nucl. Phys. B498, 28 (1997); H. Veltman, Z. Phys. C 62, 35 (1994); A. R. Bohm and N. L. Harshman, Nucl. Phys. B581, 581 (2000).

[3] M. Passera and A. Sirlin, Phys. Rev. Lett. 77, 4146 (1996).

[4] B. A. Kniehl and A. Sirlin, Phys. Rev. Lett. 81, 1373 (1998); Phys. Lett. B 440, 136 (1998).

[5] P. Gambino and P. A. Grassi, Phys. Rev. D 62, 076002 (2000).

[6] B. A. Kniehl, Nucl. Phys. B357, 439 (1991); T. Bhattacharya and S. Willenbrock, Phys. Rev. D 47, 4022 (1993); B. A. Kniehl, C. P. Palisoc, and A. Sirlin, Nucl. Phys. B591, 269 (2000).

[7] M. Passera and A. Sirlin, Phys. Rev. D 58, 113010 (1998). 
[8] P. A. Grassi, B. A. Kniehl, and A. Sirlin, Phys. Rev. Lett. 86, 389 (2001).

[9] W. Konetschny and W. Kummer, Nucl. Phys. B100, 106 (1975).

[10] N. K. Nielsen, Nucl. Phys. B101, 173 (1975).

[11] H. Kluberg-Stern and J. B. Zuber, Phys. Rev. D 12, 467 (1975).

[12] H. Kluberg-Stern and J. B. Zuber, Phys. Rev. D 12, 482 (1975); O. Piguet and K. Sibold, Nucl. Phys. B253, 517 (1985); J. C. Breckenridge, M. J. Lavelle, and T. G. Steele, Z. Phys. C 65, 155 (1995); R. Häußling, E. Kraus, and K. Sibold, Nucl. Phys. B539, 691 (1999); O. M. Del Cima, D. H. T. Franco, and O. Piguet, Nucl. Phys. B551, 813 (1999).

[13] W. Kummer, Eur. Phys. J. C 21, 175 (2001).

[14] J. C. Taylor, Nucl. Phys. B33, 436 (1971); A. A. Slavnov, Teor. Mat. Fiz. 10, 153 (1972) [Theor. Math. Phys. 10, 99 (1972)].

[15] A. Sirlin, Phys. Rev. D 22, 971 (1980).

[16] G. Degrassi and A. Sirlin, Nucl. Phys. B383, 73 (1992).

[17] K.-I. Aoki, Z. Hioki, R. Kawabe, M. Konuma, and T. Muta, Prog. Theor. Phys. Suppl. 73, 1 (1982); E. Kraus, Ann. Phys. (N.Y.) 262, 155 (1998); P. A. Grassi, Nucl. Phys. B560, 499 (1999). 